\title{
Una mezcla de manzanilla y anís estrella con actividad antimotilidad y antidiarreica en ratones
}

\author{
Roberto Guillermo Calva y Rodríguez
}

Facultad de Medicina, Benemérita Universidad Autónoma de Puebla, Puebla, Puebla, México

\section{Resumen}

Introducción: La diarrea es un serio problema de salud pública en México y otros países. Una alternativa ampliamente utilizada en el tratamiento de la diarrea es el uso de hierbas medicinales. Infusiones de manzanilla y anís estrella, que poseen propiedades antiinflamatorias y antimotilidad, podrían ayudar a aliviar los trastornos gastrointestinales. Objetivo: El objetivo de este estudio fue determinar el efecto de la mezcla de infusiones de anís estrella y manzanilla en la actividad gastrointestinal en ratones. Material y método: Se formaron 10 grupos con 10 ratones por grupo. Se evaluó el porcentaje de avance del carbón activado administrado a través del intestino de los animales. El modelo de diarrea fue inducido con aceite de ricino. Las infusiones se prepararon usando una mezcla con una relación 50:50 de las hierbas, y se administraron en una mezcla de 10, 20, 40 y 80 mg/kg por vía oral. Resultados: Los resultados indican que las mezclas 40 y 80 disminuyeron el porcentaje de avance del carbón activado, retrasaron la aparición de diarrea y disminuyeron el número de evacuaciones en comparación con el grupo control. Conclusiones: El estudio sugiere que la combinación de manzanilla y anís estrella se puede usar como un tratamiento antidiarreico alternativo.

\section{PALABRAS CLAVE: Manzanilla. Anís. Hierbas. Anti diarreica.}

\begin{abstract}
Introduction: Diarrhea is a serious public health problem in Mexico and other countries. An alternative widely used in the treatment of diarrhea is the use of medicinal herbs. Infusions of chamomile and star anise, which have anti-inflammatory and antimotility properties, could help alleviate gastrointestinal disorders. Objective: The objective of this study was to determine the effect of the mixture of infusions of star anise and chamomile on the gastrointestinal activity in mice. Material and method: Ten groups were formed with 10 mice per group. The percentage of advance of the activated charcoal administered through the intestine of the animals was evaluated. The model of diarrhea was induced with castor oil. The infusions were prepared using a mixture with a $50: 50$ ratio of the herbs, and were administered in a mixture of 10, 20, 40 and $80 \mathrm{mg} / \mathrm{kg}$ orally. Results: The results indicate that mixtures 40 and 80 decreased the percentage of advance of activated charcoal, delayed the onset of diarrhea and decreased the number of evacuations compared to the control group. Conclusions: The study suggests that the combination of chamomile and star anise can be used as an alternative antidiarrheal treatment.
\end{abstract}

KEY WORDS: Chamomile. Anise. Herbs. Anti diarreica.

Correspondencia:

Roberto Guillermo Calva y Rodríguez

E-mail: rcalva@gmail.com
Fecha de recepción: 16-08-2018

Fecha de aceptación: 20-08-2018

DOI: $10.24875 / G M M .18004580$
Gaceta Med Mex. 2018;154(Suppl 2): S61-S66

Disponible en PubMed www.gacetamedicademexico.com 


\section{Introducción}

La enfermedad diarreica ha sido reconocida durante mucho tiempo como una importante causa de morbilidad y mortalidad. ${ }^{1}$ Principalmente afecta a los niños. La diarrea aguda causa unos 5 a 8 millones de muertes por año. La mayoría de los casos de diarrea aguda ocurren en países subdesarrollados. ${ }^{2}$ Las enfermedades intestinales infecciosas (que incluyen diarrea) se encuentran entre las 20 principales causas de muerte en México. ${ }^{3}$

Las hierbas medicinales son una alternativa ampliamente utilizada para el tratamiento de la diarrea. Constituyen un componente indispensable de la medicina tradicional practicada en todo el mundo debido a su accesibilidad, experiencia ancestral y viabilidad económica. A pesar de la disponibilidad de un amplio espectro de enfoques farmacológicos para el tratamiento de la diarrea, la gran mayoría de las personas en los países en desarrollo dependen de hierbas medicinales para su manejo. ${ }^{4}$ La Organización Mundial de la Salud (OMS) ha alentado el estudio del tratamiento y la prevención de las enfermedades diarreicas mediante prácticas médicas tradicionales. ${ }^{5}$

Una de las plantas medicinales es la manzanilla (CH) (Matricaria chamomilla L.), que pertenece a la familia Asteraceae. ${ }^{6}$ Esta planta se usa en medicina tradicional para tratar heridas, úlceras, eccemas y otras dolencias. ${ }^{7,8} \mathrm{La} \mathrm{CH}$ ha sido valorada durante mucho tiempo como un relajante digestivo y se ha utilizado para tratar diversas alteraciones gastrointestinales, incluyendo indigestión, diarrea y vómitos., ${ }^{9,10}$ Además, se ha empleado para tratar cólicos, crup y fiebres en niños. ${ }^{11}$ Varios estudios sugieren que estos efectos protectores se deben a sus propiedades antiinflamatorias, antioxidantes y astringentes. ${ }^{11}$

El anís estrella (SN) (Illicium verum) es otra conocida medicina herbaria utilizada en muchas culturas, principalmente para tratar el cólico infantil, ${ }^{12,13}$ porque sus ingredientes activos incluyen anetol e hidrocarburos terpénicos (felandreno, limoneno, dipenteno) ${ }^{14}$ que le confieren su acción antiespasmódica. ${ }^{15}$ Recientemente se informó que la combinación de infusiones de plantas con propiedades antiespasmódicas podría provocar resultados más efectivos en el tratamiento de las enfermedades gastrointestinales. ${ }^{16}$ Sin embargo, no hay informes sobre el uso terapéutico de las infusiones mixtas de $\mathrm{CH}$ y SN para el tratamiento de los trastornos gastrointestinales.

Por lo tanto, el presente estudio tiene como objetivo evaluar la actividad gastrointestinal de la mezcla de $\mathrm{CH}$ y SN usando un modelo de ratones en vivo.
Método

\section{Material vegetal y preparación acuosa}

$\mathrm{La} \mathrm{CH}$ y el SN se cultivaron en los terrenos del campus de la Benemérita Universidad Autónoma de Puebla (BUAP), México. Las flores intactas se secaron a $40^{\circ} \mathrm{C}$, las muestras fueron identificadas por el autor y se depositó un comprobante muestra en el jardín botánico en la BUAP (archivo 16-13). Para preparar los extractos acuosos, las hojas fueron puestas a tierra, y con una infusión en buffer de agua con fosfato. La preparación se hizo justo antes de su uso y los extractos se administraron por sonda.

\section{Animales}

Se obtuvieron ratones CD1 machos albinos adultos (20-25 g de peso) del Bioterio Claude Bernard de la BUAP. Todos los procedimientos descritos en este estudio están de acuerdo con el Consejo Mexicano, la guía para el cuidado y el uso del laboratorio de animales y el comité de ética de la BUAP (VIEP-3447-2013). Los animales fueron alojados individualmente en una temperatura y en un ambiente controlando la humedad, y se mantuvieron bajo condiciones de luz controlada (ciclo de luz-oscuridad de 12:12 horas) con comida y agua a voluntad (ad libitum). Antes de los experimentos, todos los animales fueron mantenidos en ayunas 24 horas con acceso libre al agua.

\section{Probando la motilidad gastrointestinal}

Se formaron diez grupos ( $n=10$ por grupo): control negativo (solución salina isotónica), control positivo (Ioperamida, $5 \mathrm{mg} / \mathrm{kg}$ ), $\mathrm{CH}$ (40 y $80 \mathrm{mg} / \mathrm{kg}$ ), anís estrella (AS) $(40$ y $80 \mathrm{mg} / \mathrm{kg})$ y cuatro mezclas de grupos $\mathrm{CH}-\mathrm{AS}$ (mix-10, mix-20, mix-40 y mix-80). Los grupos de mezclas se trataron con una mezcla acuosa 50:50 de preparaciones de $\mathrm{CH}$ y AS a razón de 10, 20, 40 y $80 \mathrm{mg} /$ $\mathrm{kg}$. Todos los tratamientos se administraron por vía oral. Después de 30 minutos, a los animales se les administró una suspensión de goma arábiga (5\%) y carbón activado (10\%). Treinta minutos después, los animales fueron sacrificados por dislocación cervical y los intestinos fueron cuidadosamente retirados de la cavidad abdominal. Se midió el largo de los intestinos desde el píloro hasta el ciego y la distancia recorrida por el carbón activado. Se calculó la tasa de avance de la etiqueta en el intestino de los ratones de cada grupo. ${ }^{17}$ 


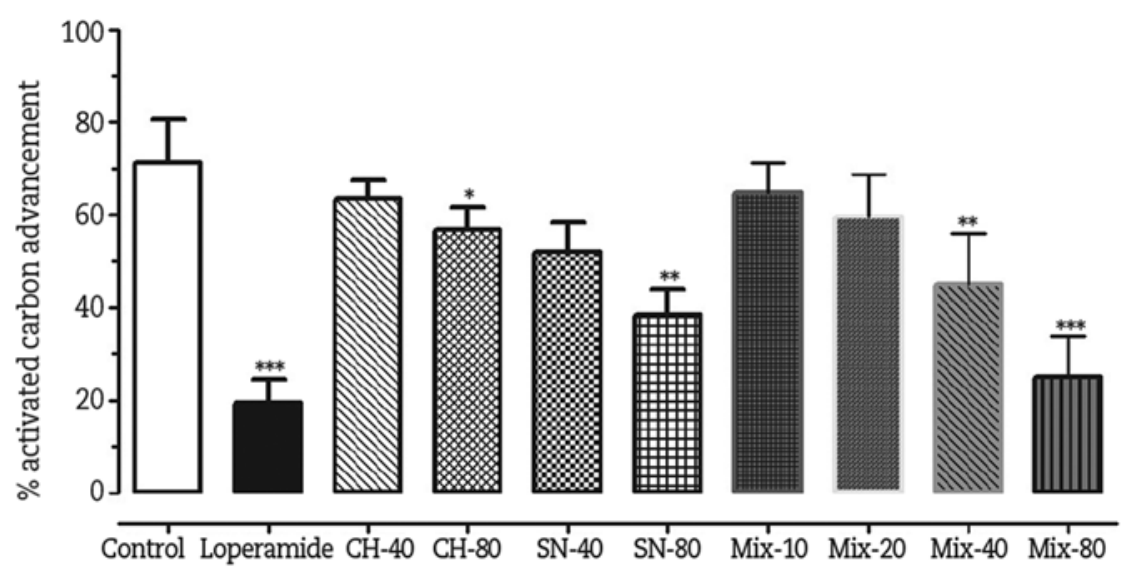

Figura 1. Efecto de la infusión de mezcla de manzanilla y anís estrella sobre la motilidad intestinal de los ratones. Se formaron diez grupos ( $n$ = 10 por grupo): control (solución salina), loperamida (5 mglkg), CH (40 y $80 \mathrm{mg} / \mathrm{kg}$ ), AS (40 y $80 \mathrm{mg} / \mathrm{kg}$ ), y mix-10, mix-20, mix-40 y mix-80. Se administró carbón y se analizó posteriormente su porcentaje de avance. Los datos trazados son la media \pm error estándar (ANOVA de una vía, posprueba de Dunnett). ${ }^{*} p<0.05 .{ }^{* *} p<0.01 .{ }^{* *} p<0.001$.

\section{Inducción del modelo de diarrea}

Se formaron diez grupos ( $\mathrm{n}=10$ por grupo): control negativo (solución salina), control positivo (loperamida, $5 \mathrm{mg} / \mathrm{kg}$ ), CH (40 y $80 \mathrm{mg} / \mathrm{kg})$, AS (40 y $80 \mathrm{mg} / \mathrm{kg}$ ) y cuatro mezclas de grupos CH-AS (mix-10, mix-20, mix40 y mix-80). Los grupos de mezclas fueron tratados con una preparación acuosa de $\mathrm{CH}$ y AS 50:50 a una dosis de 10, 20, 40 y $80 \mathrm{mg} / \mathrm{kg}$. La administración fue por vía oral para todos los tratamientos. Treinta minutos más tarde se administró aceite de ricino a cada animal. Los ratones se colocaron de forma individual en cajas, se observó el período de latencia hasta el inicio de la diarrea durante 6 horas y se contó el número de heces por grupo de animales. ${ }^{17}$

\section{Análisis estadístico}

Todos los datos se expresaron como media \pm error estándar y fueron analizados utilizando el análisis de varianza multivariante (MANOVA) seguido por la prueba de Dunnett. Se consideró significativo una probabilidad $\leq 5 \%$.

\section{Resultados}

\section{Efecto antimotilidad de la mezcla de manzanilla y té de anís estrella}

Para observar el posible efecto antimotilidad de la infusión de la mezcla $\mathrm{CH}-\mathrm{SN}$ se determinó la tasa de avance del carbón activado en el intestino delgado de ratones como parámetro para evaluar los efectos sobre la motilidad intestinal. Los resultados indican que el carbón activado del grupo de control avanzó un $71 \%$ a lo largo del intestino delgado, mientras que para el grupo tratado con loperamida avanzó un $19 \%$. El análisis comparativo entre grupos indica que el grupo al que se administró loperamida registró una disminución significativa (73\%) en el porcentaje de avance del carbón activado en comparación con el grupo de control, un hallazgo que muestra el efecto farmacológico de la loperamida en la motilidad intestinal.

Por otro lado, los grupos tratados solo con $\mathrm{CH}$ o SN en dosis de 40 y $80 \mathrm{mg} / \mathrm{kg}$ mostraron un porcentaje de avance del carbón activado del $63,57,51$ y $38 \%$, respectivamente. El análisis comparativo con el grupo control reveló que el grupo tratado únicamente con $\mathrm{CH}$ y SN en una dosis de $80 \mathrm{mg} / \mathrm{kg}$ mostró una diferencia significativa, del 20 y $46 \%$, respectivamente, pero no con la dosis de $40 \mathrm{mg} / \mathrm{kg}$. Sin embargo, hay una tendencia para disminuir el porcentaje.

Además, la administración de la mezcla de infusión $\mathrm{CH}-\mathrm{SN}$ causó una disminución en el porcentaje de avance del carbón activado, una respuesta directamente proporcional a la dosis utilizada. Los resultados informados fueron: $65 \%$ con mix-10, $60 \%$ con mix-20, $45 \%$ con mix-40 y $25 \%$ con mix80. La comparación de estos porcentajes con el grupo de control muestra una diferencia significativa en el grupo tratado con mix-40 (36\%) y mix$80(65 \%)$, lo que sugiere que las dosis de $40 \mathrm{y}$ $80 \mathrm{mg} / \mathrm{kg}$ de $\mathrm{CH}-\mathrm{SN}$ tienden a ejercer un efecto antimotilidad (Figura 1). 


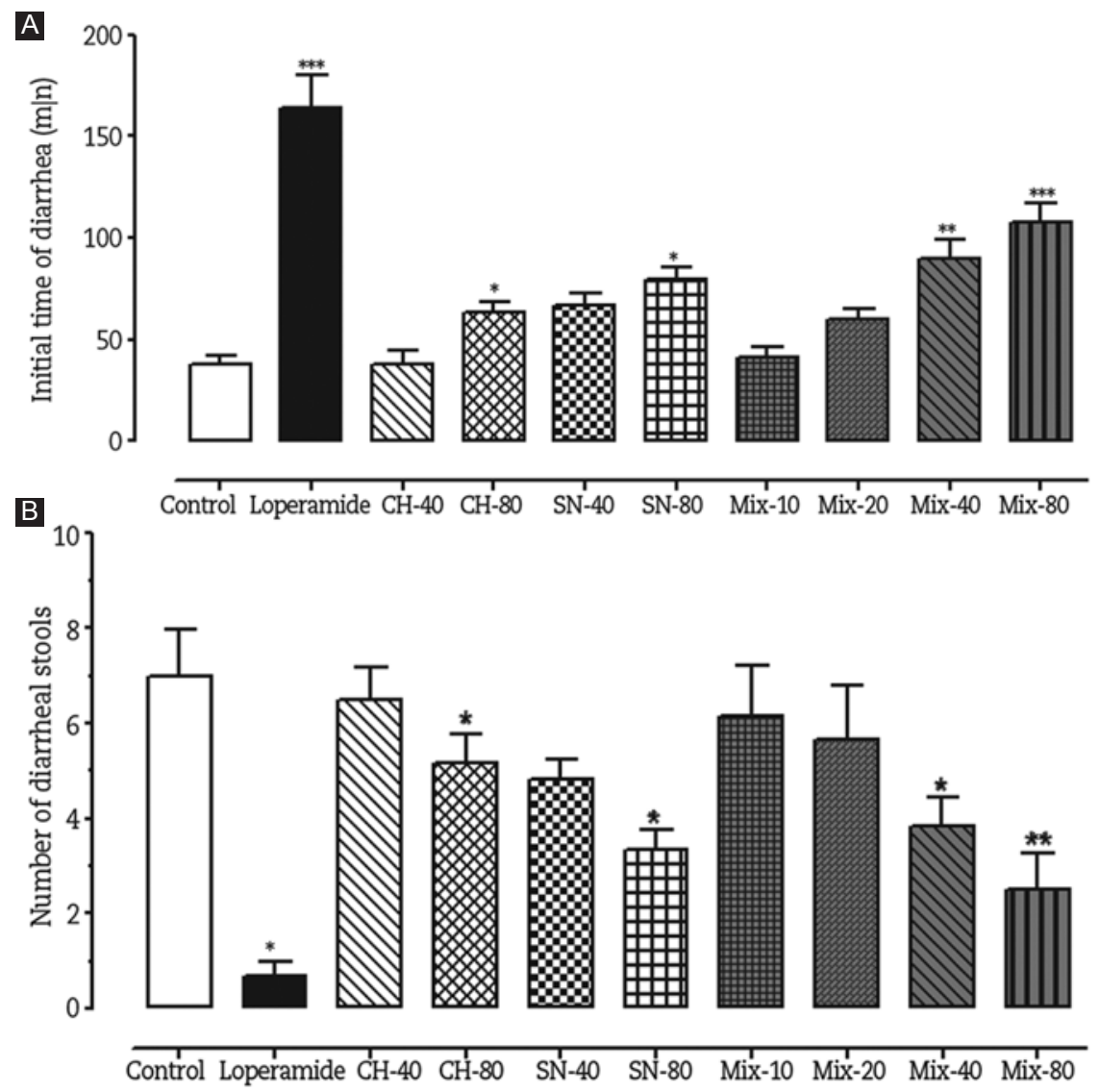

Figura 2. Efecto de la infusión de manzanilla y mezcla de anís estrella en la diarrea inducida por aceite de ricino en ratones. Los animales fueron divididos en 10 grupos ( $n=10$ por grupo): control (solución salina), loperamida (5 mg/ kg), CH (40 y $80 \mathrm{mg} / \mathrm{kg}$ ), AS (40 y $80 \mathrm{mg} / \mathrm{kg}$ ) y mix-10, mix-20, mix-40 y mix-80. Una vez que se administró el aceite de ricino, se observaron el momento del inicio de la diarrea (A) y el número de deposiciones diarreicas (B). Los datos trazados son la media \pm error estándar (ANOVA de una vía, posprueba de Dunnett). ${ }^{*} p<0.05$. ${ }^{* *} p<0.01$. ${ }^{* * *} p<0.001$.

\section{Efecto antidiarreico de la mezcla de manzanilla y té de anís estrella}

En el segundo experimento se indujo diarrea por medio de la alimentación con aceite de ricino a los ratones. Los resultados indican que el tiempo antes del inicio de la diarrea fuera más prolongado en el grupo tratado con loperamida (163 $\pm 16.2 \mathrm{~min}$ ) que en el grupo de control (36 $\pm 4.2 \mathrm{~min}$ ) (Figura $2 \mathrm{~A})$. Esto demuestra el efecto antidiarreico de la loperamida, porque la latencia para el inicio de la diarrea es tres veces mayor que en el grupo de control (Figura $2 \mathrm{~A}$ ).

La aparición de diarrea en los animales tratados solo con $\mathrm{CH}$ en dosis de $40 \mathrm{mg} / \mathrm{kg}$ ( $38.3 \pm 6.2 \mathrm{~min})$ y $80 \mathrm{mg} / \mathrm{kg}(63.5 \pm 5,02 \mathrm{~min})$ o con SN en dosis de $40 \mathrm{mg} / \mathrm{kg}(66.5 \pm 2.34 \mathrm{~min})$ y $80 \mathrm{mg} / \mathrm{kg}(79.8 \pm 5.8 \mathrm{~min})$ muestra el efecto antidiarreico por separado, mientras que las infusiones de la mezcla $\mathrm{CH}-\mathrm{SN}$ ejercían un efecto protector contra el aceite de ricino que induce la diarrea. El aumento de las proporciones de la mezcla de infusión mostró un gradual incremento en el lapso de tiempo antes del inicio de la diarrea, con resultados significativos para mix-40 (90 $\pm 8.1 \mathrm{~min})$ y mix-80 (108 $\pm 9.6 \mathrm{~min})$ en comparación con el grupo de control, y el análisis estadístico indicó una diferencia del 136 y $184 \%$, respectivamente (Figura 2 A).

En términos de gravedad de la diarrea, se observó que el número de deposiciones para el grupo de control y el grupo tratado con loperamida fue de $7 \pm 1$ y $0.6 \pm 0.33$, respectivamente. Por lo tanto, este resultado confirma la acción antidiarreica de la loperamida (91 \% frente al grupo de control). Del mismo modo, en los grupos tratados con $\mathrm{CH}$ y SN el número de deposiciones fue de $6.50 \pm 1.6$ y $5.16 \pm 0.6$ con la dosis de $40 \mathrm{mg} / \mathrm{kg}$, y $4.83 \pm 0.40$ y $3.33 \pm 0.42 \mathrm{con}$ la de $80 \mathrm{mg} / \mathrm{kg}$, respectivamente, mientras que los grupos que recibieron mix-40 $(3.8 \pm 0.6)$ y mix-80 $(2.5 \pm 0.7)$ de la mezcla $\mathrm{CH}-\mathrm{SN}$ tuvieron un menor número de deposiciones diarreicas en comparación con el grupo de control (Figura 2 B). Estos 
resultados indican que la combinación de $\mathrm{CH}-\mathrm{SN}$ tiene un efecto protector contra la diarrea inducida por aceite de ricino en ratones.

\section{Discusión}

Con sus propiedades curativas, las plantas siguen siendo el más conocido y accesible recurso para una gran proporción de la población mexicana, lo que explica el reconocimiento dado por la OMS a su gran valor como terapia medicinal en esquemas de salud públicos. Hay una evidencia científica muy fuerte relacionada con la eficacia de la medicina herbal. En México se ha atribuido propiedades medicinales a unas 4000 especies de plantas ( $15 \%$ de la flora total). Este número coincide con informes de varias regiones del mundo por especialistas en el campo, que estiman que uno de cada siete tipos de plantas posee alguna propiedad curativa. ${ }^{18}$ Desde la Antigüedad, las plantas se han utilizado para desarrollar fármacos, aromatizantes y aceites aromáticos, entre otras cosas. Las habilidades utilizadas en estos procesos han sido pasadas de una generación a otra, y actualmente son reconocidas y tema de investigación significativa en diversas disciplinas, como la química, la biología, la botánica y la farmacología. Notablemente, los hallazgos a lo largo del tiempo han revelado una gran variedad de propiedades terapéuticas, como la antimotilidad y la antidiarreica, de algunas plantas.

Los mecanismos fisiopatológicos de la diarrea incluyen aumento de la motilidad intestinal, trastornos del aumento de la secreción intestinal y procesos inflamatorios gastrointestinales. Los principales procesos implicados en la diarrea aguda son el exceso de secreción de la mucosa en respuesta a las toxinas producidas por microbios dentro de la luz intestinal y la alteración de la mucosa gastrointestinal producida por un subyacente proceso inflamatorio que altera la permeabilidad y hace que la capacidad de absorción del exudado disminuya. ${ }^{19}$ Las drogas y los productos naturales que son utilizados para atenuar la diarrea aguda ejercen sus efectos sobre dichos procesos.

$\mathrm{La} \mathrm{CH}$ y el SN son dos productos naturales que ejercen un efecto antimotilidad y modulador de la diarrea. $\mathrm{La} \mathrm{CH}$ se ha utilizado por siglos como una planta medicinal por sus propiedades antiinflamatorias y analgésicas..$^{20}$ Se consume en forma de té y ha sido aprobada en Alemania para la gestión de diversas enfermedades inflamatorias del tracto gastrointestinal, así como para aplicación tópica en el tratamiento de diversos trastornos inflamatorios de la piel y las superficies de la mucosa, como la cavidad oral y el área anogenital. ${ }^{21}$ Varios constituyentes de la $\mathrm{CH}$, como apigenina 7-O-glucósido, luteolina, compuestos terpénicos, camazuleno, (-)- $\alpha$-bisabolol, patuletina, quercetina, miricetina y rutina, se han estudiado con respecto a sus actividades antiinflamatorias. De estos, el camazuleno, el $\alpha$-bisabolol y la apigenina han demostrado poseer la mayor actividad antiinflamatoria contra agentes proinflamatorios..$^{20}$

EI SN tiene actividad carminativa, antiespasmódica, antiséptica, antimicrobiana y antidiarreica, y se usa para tratar los cólicos y como tranquilizante. Los investigadores atribuyen estos efectos a la presencia de dos derivados de la cumarina: 7-hidroxicumarina y 7-metoxi-cumarina. ${ }^{15}$ Sin embargo, el SN también contiene un dicicloéter y anetol, y ambos tienen actividades antimotilidad. El consumo de grandes cantidades de té de SN (dosis letal promedio [LD50] $4 \mathrm{~g} / \mathrm{kg}$ por vía oral en el ratón) puede causar neurotoxicidad y hepatotoxicidad. La dosis de SN utilizada en este estudio varió entre 10 y $80 \mathrm{mg} / \mathrm{kg}$, que está muy por debajo de la LD50.

Evidencia bibliográfica y los hallazgos de este estudio sugieren que la combinación de $\mathrm{CH}$ y SN puede ser más eficaz en la reducción de la motilidad y la actividad diarreica que los tratamientos convencionalmente utilizados en medicina.

La loperamida es una butiramida derivada de la piperidina con actividad del receptor mu, y es un fármaco antidiarreico activo por vía oral. ${ }^{22}$ Este estudio confirmó los efectos de antimotilidad de la loperamida por medio de un menor progreso del carbón activado a través del tracto intestinal que el encontrado en los controles. Sairam, et al. ${ }^{16}$ mostraron en 2003 resultados similares al comparar el efecto antidiarreico del mango (Mangifera indica) usando loperamida como control positivo en ratones.

La acción antimotilidad de diferentes mezclas de $\mathrm{CH}$ y SN se demostró utilizando la movilidad del carbón activado en el intestino, como inicialmente propusieron Ahmed, et al. ${ }^{23}$ y Medha, et al. ${ }^{24}$ para evaluar la contracción intestinal causada por plantas y Desmostachya tinctorum bipinnata. Los resultados de este estudio muestran que el uso de mix-40 y mix-80 reduce eficientemente la motilidad causada por el carbón activado en comparación con el grupo de control, así como en los grupos tratados con $\mathrm{CH}$ o $\mathrm{SN}$ solamente (Figura 1). Esto sugiere que la combinación de ambas infusiones de hierbas reduce la motilidad, posiblemente a través de una acción activa aditiva de ingredientes activos presentes, pero se requieren más estudios para probar esta hipótesis en humanos. 
El aceite de ricino en contacto con el ácido liberado intraluminal y las lipasas rinoleicas irrita la mucosa. El resultado es una alteración de la membrana celular debido al agua y la secreción de electrolitos, aumentando significativamente la presencia de prostaglandinas en las células gastrointestinales..$^{23} \mathrm{En}$ este estudio del efecto antidiarreico de la mezcla de $\mathrm{CH}$ y SN, los resultados mostraron que dicha mezcla disminuyó la diarrea inducida por el aceite de ricino en ratones. En este sentido, la administración de mix40 y mix-80 condujo a un retraso del inicio de la diarrea causada por el aceite de ricino, y en consecuencia el número de deposiciones diarreicas fue menor en los ratones tratados que en los del grupo de control, de modo similar a lo reportado por Romero, et al. en su estudio. ${ }^{17}$

Este efecto protector es probablemente debido a los componentes de las dos infusiones, que contienen flavonoides y glucósidos, entre otros anetoles inflamatorios, y juntos reducen el efecto nocivo del ácido ricinoleico en la mucosa, y por lo tanto la diarrea no ocurre en una extensión tan aguda. ${ }^{25}$

En conclusión, estos resultados muestran por primera vez que la mezcla de $\mathrm{CH}$ y SN (mix-40 y mix-80) ejerce un efecto antimotilidad y disminuye la diarrea inducida en ratones. Los resultados sugieren que el uso de esta combinación de infusiones de hierbas para tratar y aliviar los problemas gastrointestinales es eficiente.

\section{Bibliografía}

1. Snyder JD, Merson MH. The magnitude of the global problem of acute diarrhea disease: Are view of active surveillance of data. Bull. World Health Organ.1982;60:605-13.

2. Khan M, Khan N, Qasmi I, Zafar G. Protective effect of Arque-Ajeebonacute experimental diarrhoea in rats. BMC Complement Altern. 2004;4:8-12.
3. Programa Nacional de Salud 2007-2012. Por un México sano: construyendo alianzas por una mejor salud. México DF: Secretaria de Salud; 2007. p. 25-6.

4. Afroz S, Alamgir M, Khan MTH, Jabbar S, Nahar N, Choudhuri MSK. Anti diarrhoeal activity of the ethanol extract of Paederiafoetida $L$ inn. (Rubiaceae). J Ethno Pharmacol. 2006;105:125-30.

5. Atta AH, Mouneir SM. Anti diarrhoeal activity of some Egyptian medicinal plant extracts. J Ethnopharmacol. 2004;92:303-9.

6. Koehn FE, Carter GT. The evolving role of natural products in drug discovery. Nat Rev Drug Discov. 2005;4:206-20.

7. Rombi M. Cento piante medicinali. Bergamo, Italy: Nuovo Insttuto d'Arti Grafiche; 1993. p. 63-5.

8. Awang-Dennis VC. The herbs of choice: the therapeutic use of phytomedicinals. New York: CRC Press; 2006. p. 292.

9. Sakai $\mathrm{H}$, Misawa M. Effect of sodium azulenes sulfonate on capsaicin-induced pharyngitis in rats. Basic Clin Pharmacol Toxicol. 2005;6:54-5.

10. Crotteau CA, Wright ST, Eglash A. Clinical inquiries; what is the best treatment for infants with colic? J. Fam.Pract. 2006;55:634-6.

11. Pena D, Montes de Oca N, Rojas S. Anti-inflammatory and anti-diarrheica activity of Isocarpha cubana Blake. Pharmacology on line. 2006; 3:744-9.

12. Ize-Ludlow D, Ragone S, Bruck I, Bernstein J, Duchowny M, Pena B. Neurotoxicities in infants seen with the consumption of star anise tea. Pediatrics. 2004;114:653-6.

13. Rojas-Galarza R, Porras-A J, Li-S A. Intoxicación por anís estrella (Illicium verum): a propósito de un caso o de varios casos. Rev Peru Pediatr. 2005;58:38-41.

14. Ramos-Montes de Oca M. En el rincón de su jardín remedios caseros. Pátzcuaro Michoacán México: Morevallado Editores; 2008. p. 49-50.

15. Hall V, Rocha M, Rodríguez E. Plantas medicinales. Vol II. CIMED; 2002

16. Sairam K, Hemalatha S, Ashok K, Srinivasan T, Ganesh J, Shankar M, et al. Evaluation of anti diarrhoeal activity in seed extracts of Mangifera indica. J Ethnopharmacol. 2003;84:11-5.

17. Romero MA, Dávalos HN, Astudillo-Vázquez A. Actividad gastrointestinal del fruto de Cydonia oblonga miller. Rev Latinoamer Quim. 2009; $37: 115-21$.

18. Mantilla-Holguin J. Cultivo ecológico de plantas medicinales y aromáticas: ampliando las perspectivas económicas en los Andes. LEISA Rev Agroecología. 2005;21:33-6.

19. Riveron Corteguera R. Fisiopatología de la diarrea aguda. Rev Cubana Pediatr. 1999;71:96-115.

20. McKay DL, Blumberg JB. A review of the bioactivity and potential health benefits of chamomile tea (Matricaria recutita L.). Phytother Res. 2006;20:519-30

21. Ross SM. Chamomile: a spoonful of medicine. Holistic Nursing Practice. 2008;22:56-7.

22. Brunton LB, Lazo JS, Parker KL. Goodman \& Gilman's The pharmacological basis of therapeutics. $11^{\text {th }}$ ed. New York, NY: McGraw-Hill; 2005. p. 607-29.

23. Ahmed K, Hassane M, Abderrahim Z. Anti-diarrhoeal activity of crude aqueous extract of Rubia tinctorum L. roots rodent. J Smooth Muscle Res. 2010;46:119-23.

24. Medha M, Hedge K, Lakshman K, Girija BS, Ashok K, Lakshmiprasanna V. Assessment of anti diarrhoeal activity of Desmostachya bipinnata L. (Poaceae) root extracts. Bol Latinoam Caribe. 2010:9:312-8.

25. Meenakshi S, Kalpana G, Zafar R, Khursheed AK, Tariq BV. Bio available constituents/metabolites of pomegranate (Punicagranatum $\mathrm{L}$ ) preferentially inhibit COX2 activity ex vivo and IL-1 beta-induced PGE2 production in human chondrocytes in vitro. J Inflamm. 2008;5:1-9. 